\title{
Vesicoenteric fistula caused by bladder diverticulitis: MRI Findings
}

\author{
Ihsan Yuce ${ }^{1}$, Hayri Ogul2, Suat Eren², Ummugulsum Bayraktutan², Mecit Kantarci², Ahmet Yalcin \\ ${ }^{1}$ Department of Radiology, Erzurum Regional Training and Research Hospital, Erzurum, Turkey; \\ ${ }^{2}$ Department of Radiology, Ataturk University Medical Faculty, Erzurum, Turkey
}

\section{CASE REPORT}

The images presented below were from a man, at the age of 80, who was admitted to hospital with recurrent urinary tract infection. Magnetic resonance imaging (MRI) was performed after initial examination. Conventional MRI showed multiple diverticulum in bladder and one of them had contact sigmoid colon via fistula tract (Figure-1). Diffusion weighted images (DWI) showed restricted diffusion in a few diverticulum (Figure-2). Sagittal T2 weighted MR image shows diverticulum and bladder (Figure-3). Sistoscopic evaluation confirmed imaging findings. Pathological findings were in favor of diverticulitis and exclude malignancy.

Vesicoenteric fistulas are generally the consequences of inflammatory processes of the bowel, mainly colonic diverticulitis; colorectal cancer and, more rarely, carcinoma of the bladder; trauma; and iatrogenic fistulas between bladder and sigmoid colon as a result of prostatic surgery (1). In a study, Alapont Pérez et al. showed acquired vesicoenteric fistula in 23 patients: 48\% of these were of inflammatory etiology, 35\% were caused by neoplasms and 17\% were iatrogenic (2). Bladder diverticula are usually asymptomatic and found incidentally. Large diverticula may present with symptoms including hematuria, urinary tract infection, urinary retention, neoplasm formation, or even acute abdomen due to rupture (3). Conventional MRI with DWI is an important imaging modality for evaluation malignancies and inflammatory conditions.

Figure 1 - Enterovesical fistula secondary to diverticulitis. T1-weighted axial MR images (A-B) with intravenous contrast medium shows thick-walled diverticulum (D) and fistula track (arrow) between diverticulum (D) and bowel (B). T2-weighted axial MR images (C-D) shows bladder diverticulum (D) and large fistula track (arrow) between diverticulum (D) and bowel (B). Asterisk: bladder.
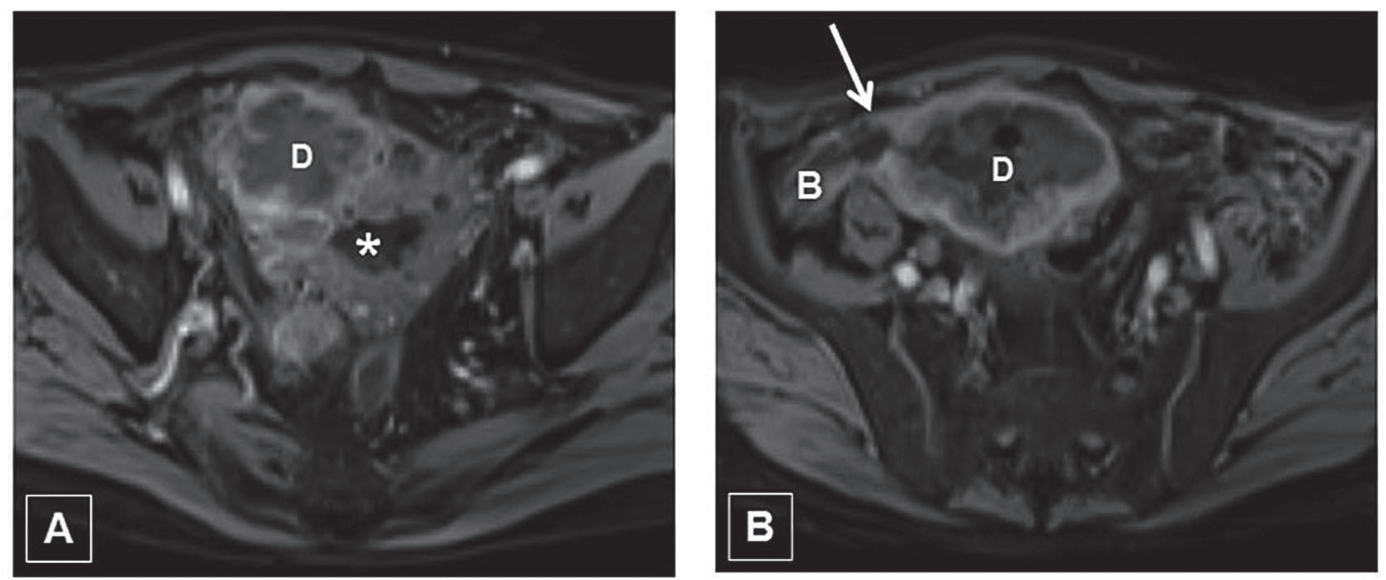
Figure 2 - Axial T2-weighted MR image (A) shows multiple bladder diverticula (D) and pseudodiverticula (arrowheads). Axial ADC image (B) shows that the diverticula (D) have a low ADC value. Asterisk: bladder.

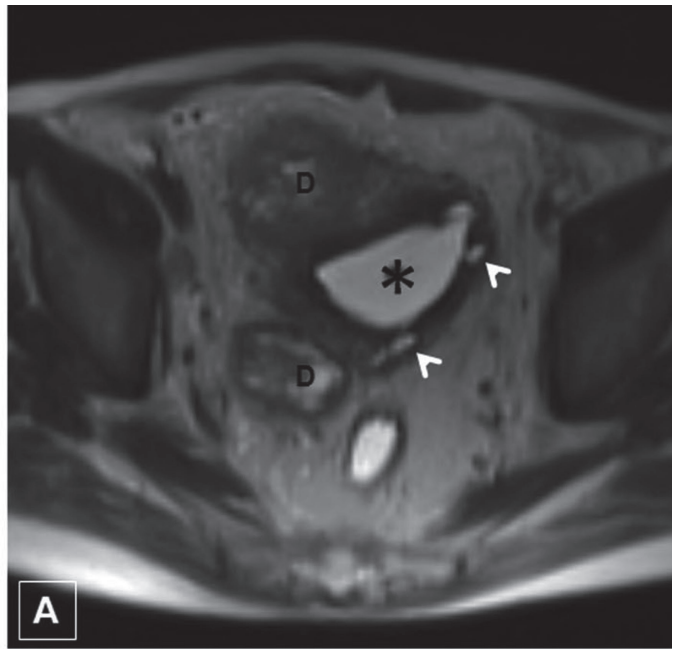

Figure 3 - Sagittal T2 weighted image shows diverticulum (D) and bladder (asterisk). P: prostate.

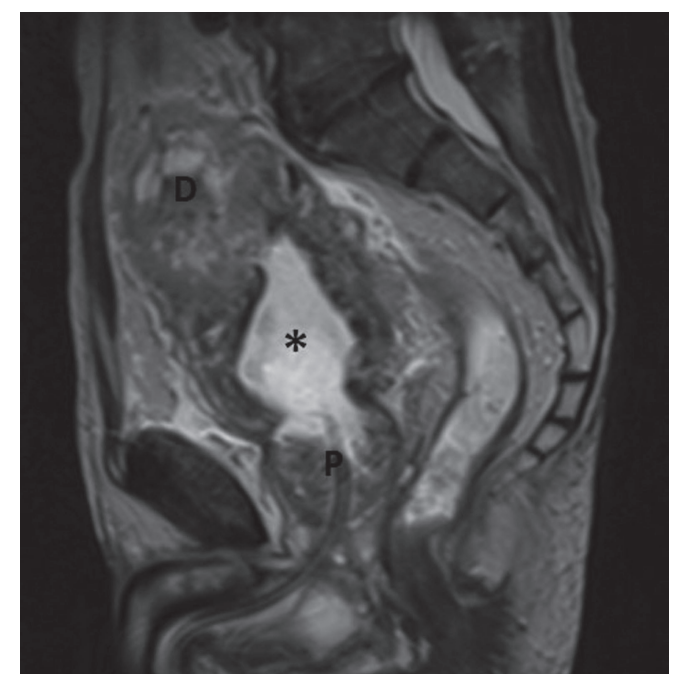

\section{ARTICLE INFO}

Int Braz J Urol. 2014; 598-9

Submitted for publication:

October 29, 2013

Accepted after revision:

June 01, 2014

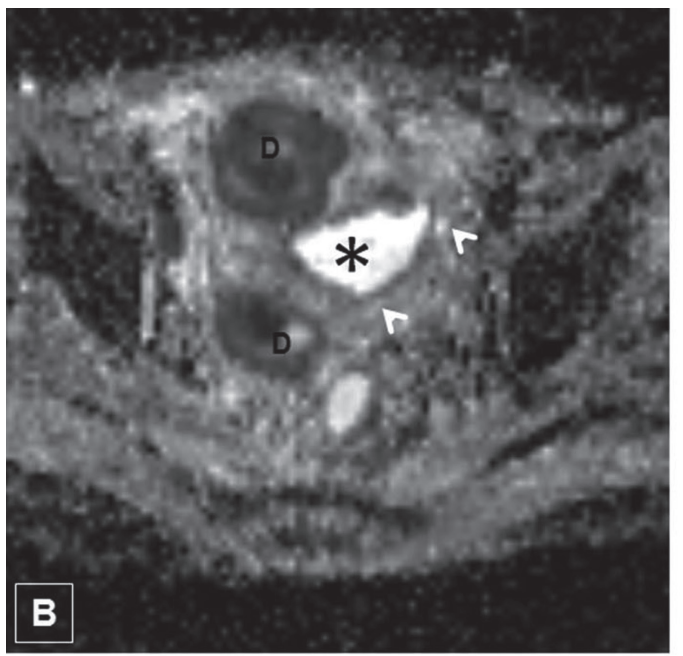

\section{REFERENCES}

1. Melekos MD, Asbach HW, Barbalias GA. Vesical diverticula: etiology, diagnosis, tumorigenesis, and treatment. Analysis of 74 cases. Urology. 1987;30:453-7.

2. Alapont Pérez FM, Gil Salom M, Esclapez Valero JP, Marti Obiol R, Santamaría Meseguer J, Rafie Mazketly W, et al. Acquired enterovesical fistulas. Arch Esp Urol. 1994;47:973-7.

3. Mićić S, Ilić V. Incidence of neoplasm in vesical diverticula. J Urol. 1983;129:734-5. 